\title{
Electrospinning of Gelatin Functionalized with Silver Nanoparticles for Nanofiber Fabrication
}

\author{
Marwa A. Oraby ${ }^{1 *}$, Ahmed I. Waley ${ }^{2}$, Ahmed I. El-Dewany ${ }^{3}$, Ebtesam A. Saad ${ }^{4}$, \\ Bothaina M. Abd El-Hady ${ }^{1}$ \\ ${ }^{1}$ Biomaterials Department, National Research Center, Giza, Egypt \\ ${ }^{2}$ Textile Department, National Research Center, Giza, Egypt \\ ${ }^{3}$ Natural Products Department, National Research Center, Giza, Egypt \\ ${ }^{4}$ Chemistry Department, Faculty of Science, Ain Shams University, Cairo, Egypt \\ Email: ${ }^{*}$ shm oraby@yahoo.com
}

Received January 24, 2013; revised February 24, 2013; accepted March 3, 2013

Copyright (C) 2013 Marwa A. Oraby et al. This is an open access article distributed under the Creative Commons Attribution License, which permits unrestricted use, distribution, and reproduction in any medium, provided the original work is properly cited.

\begin{abstract}
The present paper deals with gelatin nanofibres functionalized with silver nanoparticles, prepared by electrospinning using solutions of gelatin mixed with silver nitrate $\left(\mathrm{AgNO}_{3}\right)$. As a common solvent for gelatin and silver nitrate $\left(\mathrm{AgNO}_{3}\right)$, a mixture of acetic acid and water $(70: 30 \mathrm{v} / \mathrm{v})$ was selected. In this system, acetic acid was used as a solvent for gelatin, and at the same time reducing agent for silver ions in solution. Silver nanoparticles (nAg) were stabilized through a mechanism that involves an interaction of the oxygen atoms of the carbonyl groups of gelatin. The viscosity and the conductivity of the gelatinous solutions were found to increase with the solution concentration. There is an observed decrease in the viscosity of the $\mathrm{nAg}$ containing gelatin solutions with the aging time increasing, whereas the conductivity of the $\mathrm{AgNO}_{3}$ - containing gelatin solutions was greater than that of the base gelatin solution. The gelatin nanofibres functionalized with silver nanoparticles were characterized by transmission electron microscopy (TEM), scanning electron microscopy (SEM), X-ray diffraction (XRD) and antimicrobial test. The results of investigations by TEM and XRD confirmed the presence of silver nanoparticles with diameters in the range of $(2-10 \mathrm{~nm})$, uniformly distributed over the surface of smooth nanofibres with an average diameter of $70 \mathrm{~nm}$. The release of silver ions from both the 2and 4-hrs crosslinked nAg containing gelatin fiber mats by a total immersion method in buffer and distilled water occurred rapidly during the first 60 minutes, and increased gradually afterwards. Lastly, the tests demonstrated that gelatin/Ag nanofibers have a good antimicrobial activity against some common bacteria found on burned wounds. The antibacterial activity of these materials was greatest against Staphylococcus aureus, followed by Escherichia coli, and Pseudomonas aeroginosa $\approx$ Candida albicans.
\end{abstract}

Keywords: Electrospinning; Gelatin; Silver; Nanofibers; Nanoparticles; Antimicrobial Activity

\section{Introduction}

One of the very important tissues for human health is the skin, which plays the role of an interface between body and environment, and acts as a protective barrier against physical, chemical and biological aggressions. Skin is actually a complex systems involved in the processes of absorption and excretion, showing a selective permeability for some important substances in human metabolism [1].

The currently increasing incidence of skin diseases caused a significant demand for skin replacement materials which in many cases cannot be assured from natural

"Corresponding author. sources. It also increased the demand for skin regrowth substrates used in treating skin lesions, which stimulated tissue engineering to develop new biodegradable materials. These materials can promote adhesion, development and migration of fibroblasts which are necessary for a good quality reepithelization. Skin substitutes used today are expensive and have limitations concerning the biocompatibility, making their use sometimes risky. Various technologies have been developed to produce biomaterials used for skin lesions treatment of wound dressings. One of the very interesting nanotechnologies investigated now is polymer solutions spinning in an electrostatic field (electrospinning), which can be applied to solutions of various natural and synthetic polymers, such as gelatin, 
collagen, chitosan, fibrinogen, silk, polylactic acid, hyaluronic acid, obtaining non-woven nanofibres membrane with thicknesses in a range that goes from a few tens of nanometers to over one thousands of nanometers [2]. This type of three-dimensional structure of nanofibres prepared by electrospinning resembles very well with the human extracellular matrix, which explains the great interest manifested today for this nano-technology. The morphology of the electrospun (e-spun) fibers depends on a number of factors, such as solution properties (e.g., concentration, viscosity, conductivity, surface tension, etc.), processing conditions (e.g., electrical potential, collection distance, etc.), and ambient conditions (e.g., temperature, humidity, etc.) [3].

Natural biopolymers have high viscosity and very low solubility in common organic solvents, which makes them difficult to process by solution spinning in an electrostatic field. Natural polymers dissolve well in 1,1,1,3,3,3-hexafluoro-2-propanol and trifluoroacetic acid [4-7], which are toxic and expensive solvents, features that limit their use in applications on an industrial scale.

Due to its natural abundance and inherent biodegradability in physiological environments, gelatin is widely used in food, cosmetic, pharmaceutical and medical applications [8]. Depending on its usage, gelatin can be fabricated in many forms, e.g., films [9], micro- or nanoparticles [10,11], and dense or porous hydrogels [12-14]. Gelatin in the form of micro- and nanofibers can also be fabricated by gel and electrospinning techniques, respectively [15]. Owing to the uniqueness of the e-spun fibers (e.g., high surface area to mass or volume ratio; high porosity of the e-spun fiber mat; and flexibility for surface functionalization), e-spun gelatin fibers are of interest here. Suitable solvents for preparing an electrospinnable gelatin solution are 2,2,2-trifluoroethanol (TFE), formic acid, 1,1,1,3,3,3-hexafluoro-2-propanol (HFP), and acetic acid.

Because of the inherent properties of gelatin and the unique characteristics of the e-spun fibers, e-spun gelatin fibers are ideal materials to be used as scaffolds for cell and tissue culture, carriers for topical/transdermal delivery of drugs, and wound dressings.

Bacterial colonization and subsequent infection can interfere with the wound healing process by producing various substances (e.g., toxins, proteases and proinflammatory molecules) which may cause an excessive and prolonged inflammatory response of the host tissues [16-19].

Idealantimicrobial dressings should have a number of key attributes, including provision of a moist environment to enhance healing [20], and broad-spectrum antimicrobial activity, including activity against antibioticresistant bacteria [e.g., methicillin-resistant Staphylococ- cus aureus (MRSA)] [16-19]. A recent resurgence of the use of silver-based dressings has been ascribed to their broadspectrum antibacterial activity, as well as to a less possibility for inducing bacterial resistance than antibiotics $[17,18]$.

Silver nanoparticles (nAg) are well known for strong antibacterial properties and no harm on human cells [21]. Recently, considerable attention has been paid to the incorporation of silver nanoparticles into ultrathin fibers for many important applications, such as using wound dressing materials in medical fields [22]. Some researches electrospun silver nanoparticles (nAg) containing nanofibers by adding metallic salts (mainly silver nitrate) into the polymer solutions [23].

Some polymers have been successfully used for the synthesis of metal nanoparticles. The binding interaction between polymer and the metal nanoparticles, polymer stabilizes the metal nanoparticles-polymer composite, and hence, the nanoparticles attached to the polymer chains will be dispersed in the solution when the composite dissolves. Therefore, it is possible to prepare the metal nanoparticles containing nanofibers via electrospinningthe solution of the metal nanoparticle- polymer composite, if the size of nanoparticles is small enough.

In the present study, mats of gelatin fibers containing $\mathrm{nAg}$ were prepared by electrospinning. The proposed use of these e-spun fiber mats is as wound dressing pads. Glutraldhyde (GTA) vapor was used to improve the stability of the fiber mats in a moist environment. The nAg-containing gelatin fiber mats were characterized for their release characteristics of the as-loaded silver, as well as for their antibacterial activity against some common bacteria found on burn wounds.

\section{Experimental Details}

\subsection{Materials}

Gelatin (from porcine skin, type A) was purchased from Sigma Aldrich.Silver nitrate $\left(\mathrm{AgNO}_{3} ; 99.9 \%\right.$ purity) with a molecular weight of 169.87 was purchased from Sisco Research Laboratories PVT.LTD (SRL). Acetic acid (96\%) $\left(\mathrm{C}_{2} \mathrm{H}_{4} \mathrm{O}_{2}\right)$ with a molecular weight of 60.05 was purchased from (Adwic) El-Nasr Pharmaceutical Chemicals Co. Glutaraldehyde, (GTA; $25 \mathrm{wt} \%$ solution in water) $\left[\mathrm{HCO}\left(\mathrm{CH}_{2}\right)_{3} \mathrm{CHO}\right]$ was purchased from Sigma Aldrich. Glycinepure lab. Chemical (99\%) with a molecular weight of 75.07 was purchased from (Adwic) El-Nasr Pharmaceutical Chemicals Co. All chemicals were of analytical reagent grade and used without further purification.

\subsection{Preparation and Characterization of Gelatin Solutions Containing Silver Nanoparticles (nAg)}

$\mathrm{AgNO}_{3}$ was first dissolved in a quantity of $70: 30 \mathrm{v} / \mathrm{v}$ gla- 
cial aceticacid/distilled water. A metered weight of gelatin powder was then added into the as-prepared $\mathrm{AgNO}_{3}$ solution. Slight stirring was used to expedite the dissolution and homogenize the solution. The concentration of the base gelatin solution was $50 \% \mathrm{w} / \mathrm{v}$ (based on the volume of the mixed solvent), and the amount of $\mathrm{AgNO}_{3}$ was $2.5 \% \mathrm{w} / \mathrm{w}$ (based on the weight of the gelatin powder). To investigate the effect of aging time on the formation of $\mathrm{nAg}$, the $\mathrm{AgNO}_{3}$-containing gelatin solution was aged in a brown bottle while being stirred for varying periods of time. Some of the aged $\mathrm{AgNO}_{3}$-containing gelatin solutions were characterized for shear viscosity and conductivity using a Brookfield DV-III ultra-programmable rheometer and a professional portable conductivity meter HC3010, trans-instruments. The presence of silver only in the form of crystallized metal nanoparticles is certified by the X-ray diffractogramme. The morphology of the electrospun fibers was observed under a Quanta FEG 250 FEI, Holanda scanning electron microscope (SEM) after gold coating by an Ion sputtering with $15 \mathrm{~mA}$ for 3.5 minutes each sample. The diametets of the as formed nAg was observed under Jeol Jem-1230 transmission electron microscope (TEM).

\subsection{Preparation of Neat and nAg-Containing E-Spun Gelatin Fiber Mats}

\subsubsection{E-Spinning}

The base gelatin solution and the $\mathrm{AgNO}_{3}$-containing gelatin solution that had been aged for $12 \mathrm{~h}$ were fabricated into fiber mats by e-spinning. Firstly, each of the as-prepared solutions was loaded into a standard 3-mL plastic syringe, the open end of which was attached to a needle, which was used as the nozzle. Apiece of aluminum (Al) sheet was placed below the capillary tip as the ground collector. Capillary was connected with a high voltage power supply (Model Glassman high voltage, NJ08829), which could generate positive DC voltages. A High-Voltage DC powersupply was used to charge the solution by attaching the emitting electrode of positive polarity to the nozzle, and the grounding one to the collecting device. An electrical potential of $20 \mathrm{kV}$ was applied across a distance of $18 \mathrm{~cm}$ between the tip of the needle and the outer surface of the collection device (i.e., collection distance).

\subsubsection{Crosslinking}

Crosslinking of both the neat and the nAg-containing gelatin fiber mats was carried out by soaking the fiber mat samples in $2 \mathrm{wt} \%$ glutaricdialdehyde (GTA/ethanol) for different time intervals. After exposure, the samples were washed with $0.2 \mathrm{M}$ glycine in order to remove the unreacted GTA.

\subsubsection{Characterization}

The neat and the nAg-containing gelatin fiber mats were examined either qualitatively or quantitatively for the size and/or the amount of both the individual fibers and the as-formed nAg using a Quanta FEG 250 FEI, Holandascanning electron microscope (SEM) and an energy dispersive X-ray (EDX), a Jeol Jem-1230 transmission electron microscope (TEM). The average diameters of both the fibers and the as-formed nAg were determined from SEM and TEM images, respectively. FT-IR measurements were performed in a FT-IR Spectrometer 1760× (Perkin-Elmer) to verify the composition of fibers from functional groups.

\subsubsection{Loading Capacity and Release Characteristics of As-Loaded Silver}

Prior to the release assay, the actual amount of silver in the uncrosslinked $\mathrm{nAg}$-containing gelatin fiber mat specimens $(2 \mathrm{~cm} \times 2 \mathrm{~cm})$ and the form of silver that was released from the specimens were needed to be determined. The in-vitro release of silver was carried out by the addition of a releasing medium (buffer, and distilled water) to attain a total volume of $5 \mathrm{ml}$.

The release characteristics of silver from the $\mathrm{nAg}$ containing gelatin fiber mats that had been soaked in the GTA solution for $2 \mathrm{hrs}$ or $4 \mathrm{hrs}$ was assessed in buffer, and distilled water as the releasing medium. The specimens cut from the fiber mat samples $(2 \mathrm{~cm} \times 2 \mathrm{~cm})$ were immersed in $5 \mathrm{ml}$ of the releasing medium at room temperature.

At a specified immersion periods ranging between 0 and $7 \mathrm{~d}$, the releasing medium was quantified for the amount of released silver, using Inductively Coupled Plasma (ICP). At each time point, the measurements were carried out in triplicates. The obtained data were carefully calculated to obtain the cumulative amount of released silver. The cumulative release profiles of silver were expressed based on either the unitweight of the specimens or the unit weight of the actual amount of silver in the specimens. The results were presented in term of cumulative release as a function of release time.

$$
\text { Cumulative amount of release }(\%)=\left(M_{t} / M_{\infty}\right) \times 100 \%
$$

Where $M_{t}$ was the amount of silver released at time $t$, the amount of silver added to electrospinning solution was regarded as $M_{\infty}$.

\subsubsection{Antibacterial Evaluation}

The antibacterial activity of the e-spun fiber mats, both from the base solution and the $12 \mathrm{~h}$-aged $\mathrm{AgNO}_{3}$-containing gelatin solution were tested against aerobic bacteria commonly found on burn wounds: e.g., E. coli (Gramnegative), Pseudomonas aeroginosa (Gram-negative), $S$. aureus (Gram-positive), and Candida albicans. The assessment was conducted based on the disc diffusion method. If inhibitory concentrations were reached, there 
would be no growth of the microbes, which could be seen as a clear zone around the disc specimens. Those were photographed for further evaluation.

\section{Results and Discussion}

\subsection{Effect of Aging Time on nAg-Containing Gelatin Solutions}

Prior to e-spinning, some of the $\mathrm{AgNO}_{3}$-containing gelatin solutions that had been aged for different time intervals were measured for their viscosity and conductivity. The observed decrease in the shear viscosity of the solutions with increasing the aging time could be a result of a considerable hydrolytic degradation of gelatin molecules. While the conductivity of the $\mathrm{AgNO}_{3}$-containing gelatin solutions was greater than that of the base gelatin solution. This could be as a result of the presence of $\mathrm{Ag}^{+}$and $\mathrm{NO}_{3}{ }^{-}$ions in the solution as shown in Figure 1 [24].

\subsection{Neat and nAg-Loaded E-Spun Gelatin Fiber Mats}

\subsubsection{Fourier-Transform Infrared Spectroscopy (FT-IR)}

In order to determine electrospun gelatin fiber structure before and after crosslinking, FT-IR was carried out on the electrospun fibers before and after crosslinking. Figure reports the FT-IR spectra of gelatin fiber mats prepared from acetic acid/water solution, and from crosslinked gelatin together.

FTIR measurements were conducted on uncrosslinked gelatin and crosslinked gelatin in order to determine whether crosslinking of the electrospun gelatin affected the gelatin structure or not (Figure 2).

The uncrosslinked electrospun gelatin had an amide I peak $(\mathrm{C}=\mathrm{O}$ stretch $)$ at $1636-1640 \mathrm{~cm}^{-1}$, amide II peak (N-H bend and C-H stretch) at $1542-1544 \mathrm{~cm}^{-1}$, amide III peak (C-N stretch plus N-H in phase bending) at 1240 $\mathrm{cm}^{-1}$ and amide A peak (N-H stretching vibration) at $3316 \mathrm{~cm}^{-1}$, which are the distinguishing features of gelatin.

The spectrum of the crosslinked gelatin is shown in detail in Figure 2. In addition to the previously mentioned peaks, a strong peak at $1450 \mathrm{~cm}^{-1}$ was observed in the crosslinked gelatin due to aldimine absorption [25]. In comparison to uncrosslinked gelatin $[25,26]$ we found that the amide II peak was changed from smooth to several small peaks. Glutaraldehydes have an aldehyde group (-CHO) that reacts with the amino group of the lysine residues of proteins [26]. The uncrosslinked and crosslinked membranes were discriminated by a slow change in color from white to yellow. The color change occurred because the aldimine linkage $(\mathrm{CH}=\mathrm{N})$ reactions took place during the crosslinking process.

The characteristic absorption of the aldimine groups
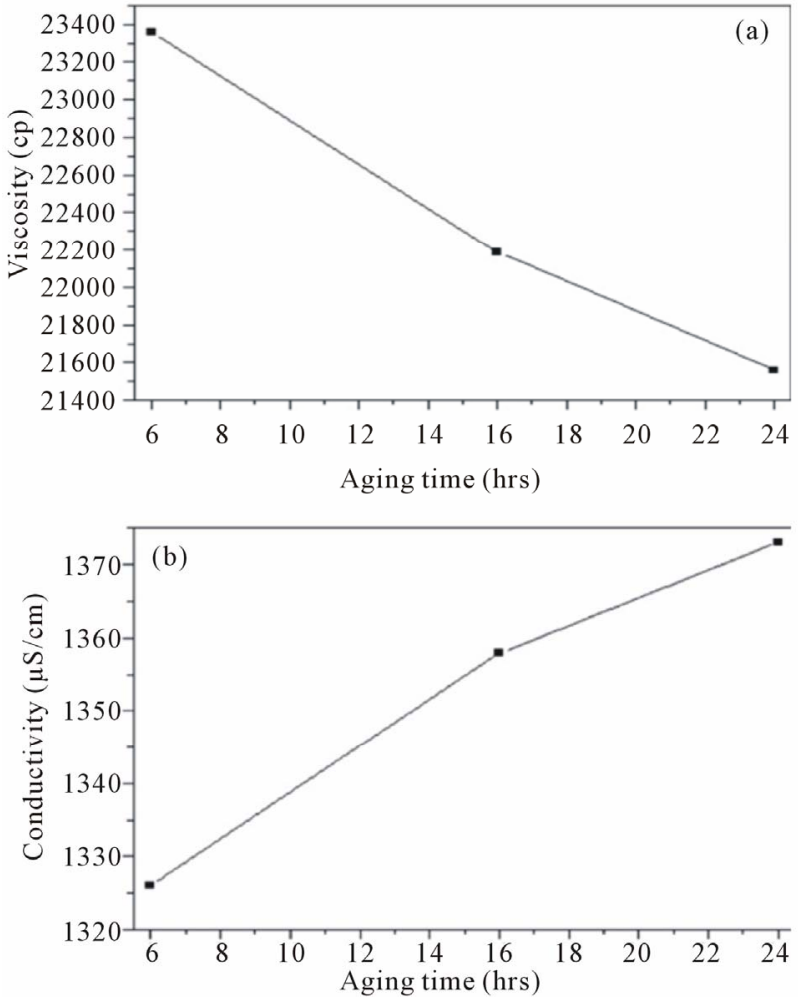

Figure 1. (a) Viscosity and (b) Conductivity of the $\mathrm{AgNO}_{3}$ containing gelatin solutions in acetic acid as a function of the aging time.

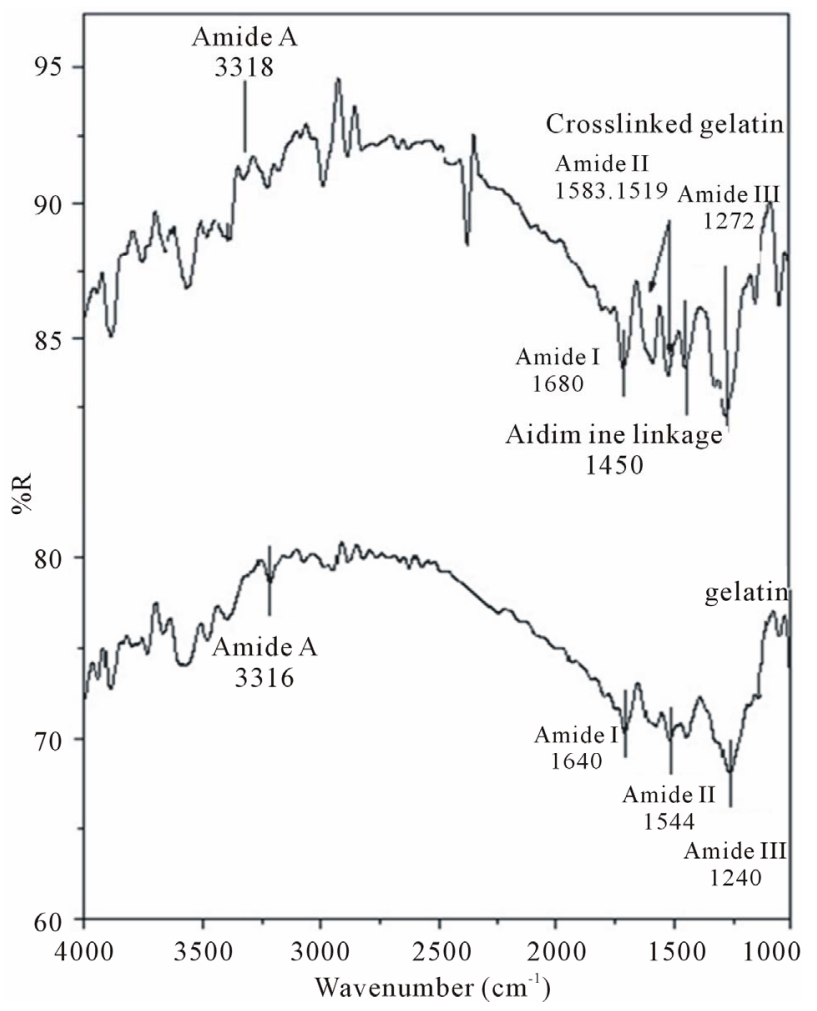

Figure 2. FT-IR of uncrosslinked gelatin and crosslinked gelatin electrospun fibers. 
occurred at $1450 \mathrm{~cm}^{-1}$. Furthermore, additional peaks were observed at $1470-1570 \mathrm{~cm}^{-1}$, which increased as the crosslinking reaction was proceeded (Figure 2). To more conclusively determine if crosslinked gelatin was successfully fabricated, we expanded the FT-IR spectra from $1100-1750 \mathrm{~cm}^{-1}$ (Figure 2). In this region of the FT-IR spectra, the amount of $\mathrm{CH}=\mathrm{N}$ groups increased [26]. Thus, both uncrosslinked and crosslinkedelectrospun mats gelatin were successfully fabricated via the electrospinning method using acetic acid/water as shown in Figure 2.

This technique proved crosslinking of gelatin using glutaraldehyde during the crosslinking process, which is an essential condition to limit the insolubility and increase the biocompatibility of gelatin for applications in the biomedical field [26].

\subsubsection{X-Ray Diffraction Analysis (XRD)}

XRD was used to confirm the success of silver nanoparticles incorporation into the electrospun gelatin. The patterns of electrospun gelatin are not crystalline and appear amorphous (not shown).The XRD pattern of electrospun gelatin fiber mats was shown in Figure 3. A broad hallow can be seen around $\left(2 \theta=22^{\circ}\right)$ in the XRD pattern, which is attributed to the diffraction peak of gelatin [27]. The above results indicate the decrease in crystallinity of silver nanoparticles. It was due to the incorporation of amorphous gelatin into electrospun fibers. The presence of silver only in the form of crystallized metal nanoparticles is certified by the X-ray diffractogramme shown in Figure 3, where the peak due to silver is clearly present. XRD pattern of the electrospun fiber mats reveals the presence of the main peaks which are characterizing the structure of silver nanoparticles according to the Powder Diffraction File (JCPDS Card No. 87-0598). The interplanerspacings $(\mathrm{d} \AA)$ of these peaks are recorded at 2.5000, 2.4249, 2.2357, 1.9997, 1.4431, 1.3866, 1.2498, and 1.2402 .

This is implies the presence of the Ag nanoparticles which are embedded in the gelatin fibers.

\subsubsection{Morphology before and after Crosslinking Treatment}

Figure 4 shows selected SEM images of e-spun fiber mats for both the neat gelatin and the 12 hrs-aged $\mathrm{AgNO}_{3}$-containing gelatin solutions. Only cross-sectionally round fibers, without the presence of beads, were obtained. The diameters of the individual fibers obtained from these solutions were in the range of $98-345$ and 154 - 390 nm, respectively. Figure 5 shows a TEM image of an e-spun fiber for the $12 \mathrm{~h}$-aged $\mathrm{AgNO}_{3}$-containing gelatin solution. Apparently, $\mathrm{nAg}$ was distributed throughout the fibers, with the diameters of those particles being $2-10 \mathrm{~nm}$. Notwithstanding, it should be

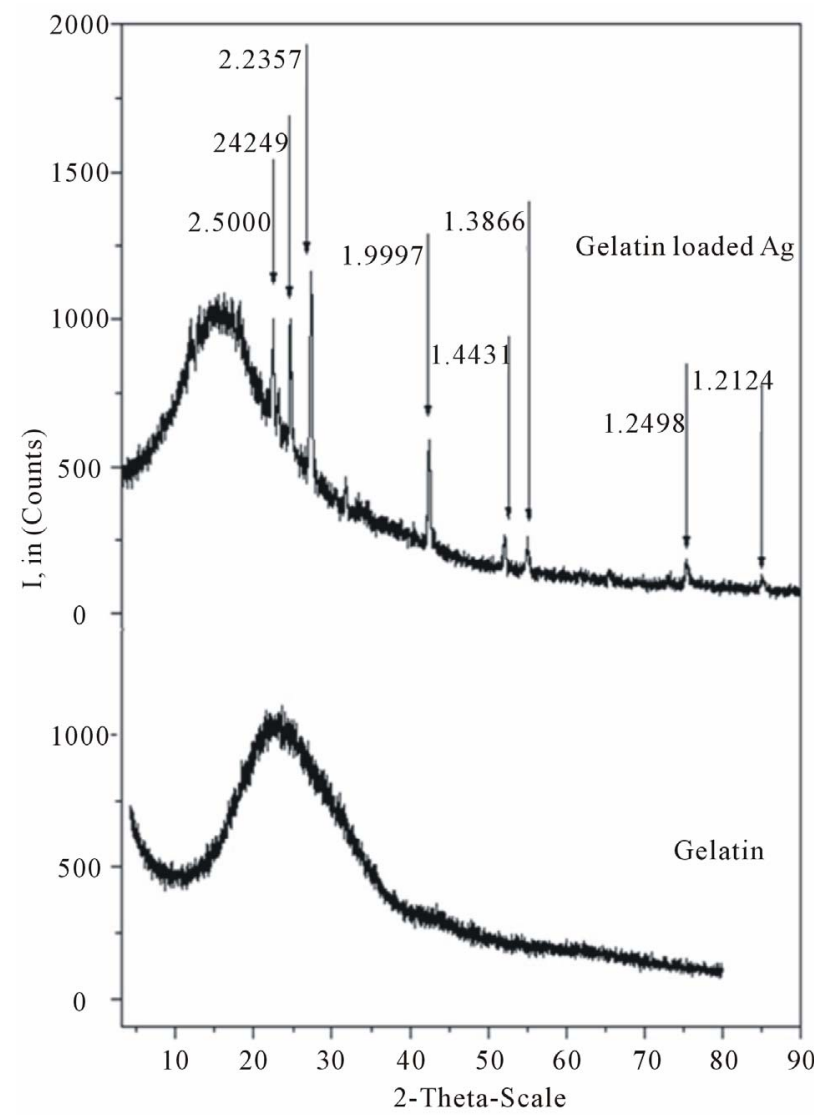

Figure 3. X-ray diffractograms of electrospun gelatin nanofibers and gelatin nanofibers functionalized with silver nanoparticles.

emphasized that, at $12 \mathrm{~h}$ of aging, silver was present both as $\mathrm{Ag}$ ions and as elemental $\mathrm{Ag}$; but for convenience, $\mathrm{nAg}$ was used loosely to denote both forms of silver that were present in the e-spun fiber. Quantitative assessment by EDX for the amount of the elemental Ag in the e-spun fiber mat from the $12 \mathrm{hrs}$ aged $\mathrm{AgNO}_{3}$-containing gelatin solution indicated a value of $0.25 \mathrm{wt} \%$.

Here, the formation of the $\mathrm{nAg}$ nuclei was postulated in order to originate due to ionic interactions between the $\mathrm{Ag}^{+}$ions and either $-\mathrm{NH}_{2}$ or $-\mathrm{COOH}$ groups, or both, on the gelatin chains, according the following schemes:

$$
\begin{gathered}
\mathrm{H}_{2} \mathrm{~N}-\mathrm{COOH}+\mathrm{AgNO}_{3} \leftrightarrow \mathrm{H}_{2} \mathrm{~N}-\mathrm{COOAg}+\mathrm{HNO}_{3} \\
\mathrm{H}_{2} \mathrm{~N}-\mathrm{COOH}+\mathrm{AgNO}_{3} \leftrightarrow \mathrm{AgHN}-\mathrm{COOH}+\mathrm{HNO}_{3}
\end{gathered}
$$

or due to simple binding between $\mathrm{Ag}^{+}$ions and lone pair electrons of $\mathrm{N}$ and $\mathrm{O}$ atoms in the $-\mathrm{NH}_{2}$ and $-\mathrm{COOH}$ groups [28] followed by the reduction of $\mathrm{Ag}^{+}$ions into nAg nuclei.

Since gelatin is water-soluble, an e-spun gelatin fiber mat can easily dissolve either partially or completely, losing its fibrous structure when coming into contact with an aqueous medium. It may partially dissolve and loose its fibrous structure upon an amount of exposure to 

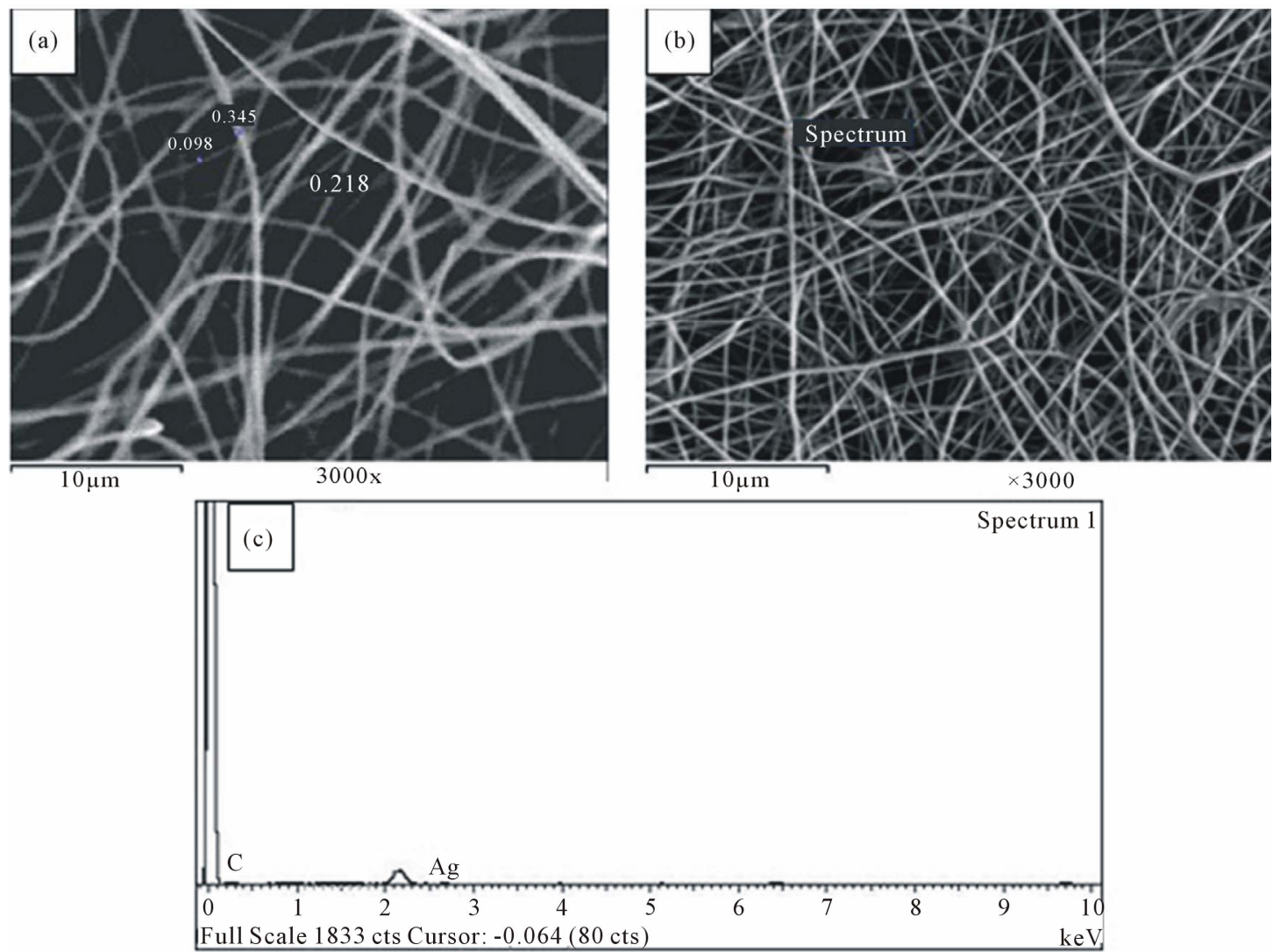

Figure 4. Selected SEM of the electrospun fiber mats from (a) The neat gelatin and (b) The AgNO $_{3}$-containing gelatin solutions that had been aged for $12 \mathrm{~h}$ at magnification 3000×; (c) SEM associated EDAX area analysis of electrospun fiber mats.
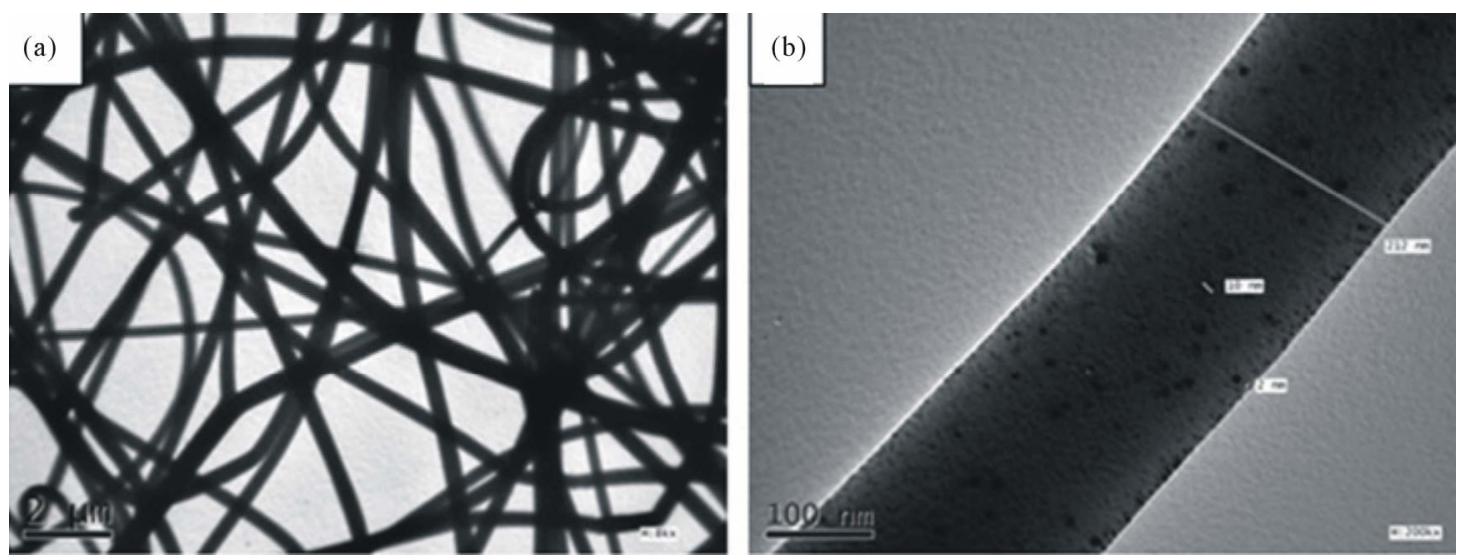

Figure 5. Selected TEM image of an electrospun fiber from the (a) Base gelatin; (b) $\mathrm{AgNO}_{3}$-containing gelatin solution that had been aged for $12 \mathrm{~h}$. The diameters of the as-formed nAg were from (2-10) $\mathrm{nm}$.

a high ambient humidity (e.g., 80\% - 90\%) for a certain period of time. To extend the use of e-spun gelatin fiber mats in applications that require exposure to an aqueous medium or high humidity, further crosslinking is necessary. Among the various chemical systems used to crosslink an e-spun gelatin fiber mat.

GTA is seemingly the most suitable, as it is economical and does not compromise the fibrous structure of the e-spun membrane. Here, the neat and the nAg-containing e-spun gelatin fiber mats were further crosslinked by GTA aqueous solution for either 2 or 4 hrs. Crosslinking treatment did not affect the amount of $\mathrm{nAg}$ in the crosslinked e-spun gelatin fiber mats [29].

Figure 6 shows selected SEM images of the e-spun fiber mats for the neat gelatin solutions after having been crosslinked with GTA for either 2 or $4 \mathrm{hrs}$. Evidently, the neat after cross-linking, changed their color from white to yellow (for the fibers mats that had been cross-linked 


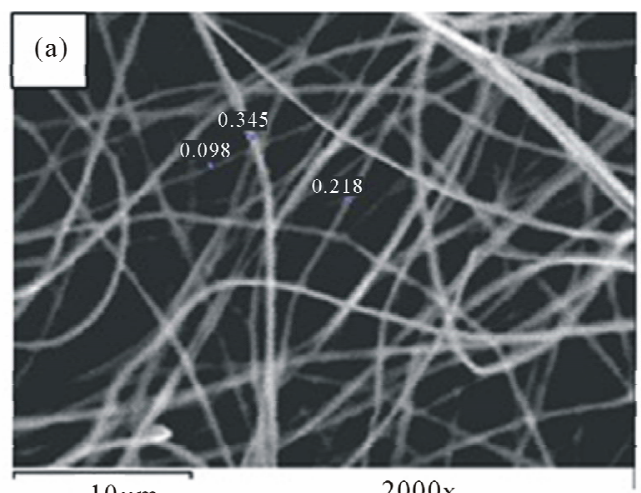

$10 \mu \mathrm{m}$

$2000 x$
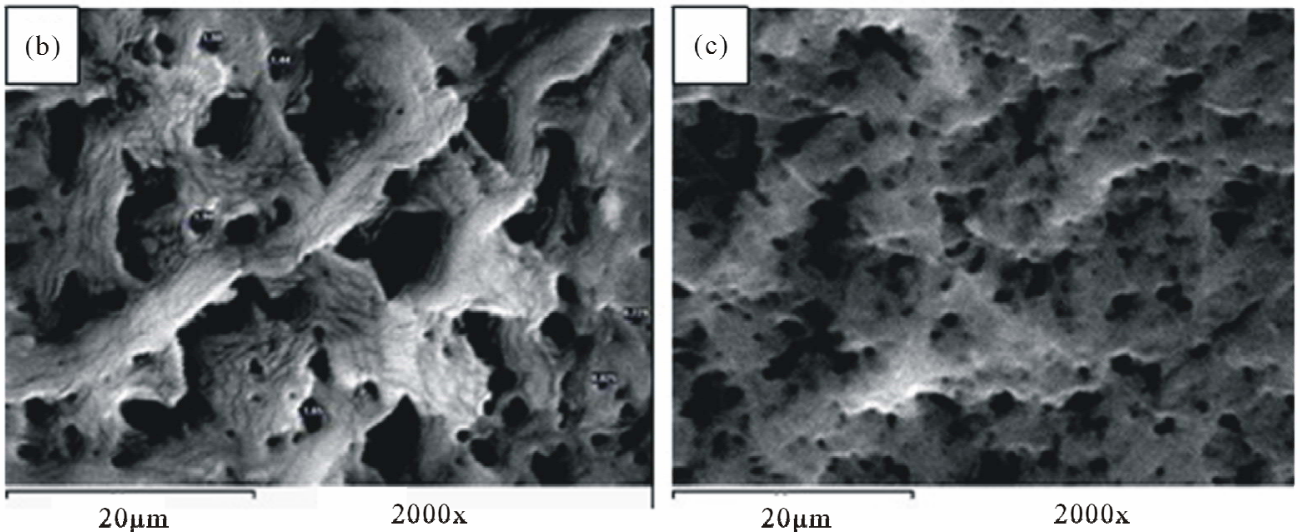

Figure 6. Morphology of the electrospun fiber mates from the $50 \mathrm{wt} \%$ gelatin solution. (a) The base gelatin solution (before crosslinking) (magnification $3000 \times$ ); (b) and (c) The neat gelatin solution that had been crosslinked with glutraldhyde (GTA) for 2 hrs, and 4 hrs (magnification $2000 \times$ ) respectively.

for $2 \mathrm{hrs}$ ) and finally to brown (for the fiber mats that had been crosslinked for $4 \mathrm{hrs}$ ). They also shrunk slightly from their original dimensions. The change in color of the gelatin upon crosslinking with GTA is caused by the formation of aldimine linkages $(-\mathrm{CH}=\mathrm{N}-)$ between the free amino groups of lysine or hydroxylysine amino acid residues of the protein and the aldehyde groups of GTA $[25,30]$.

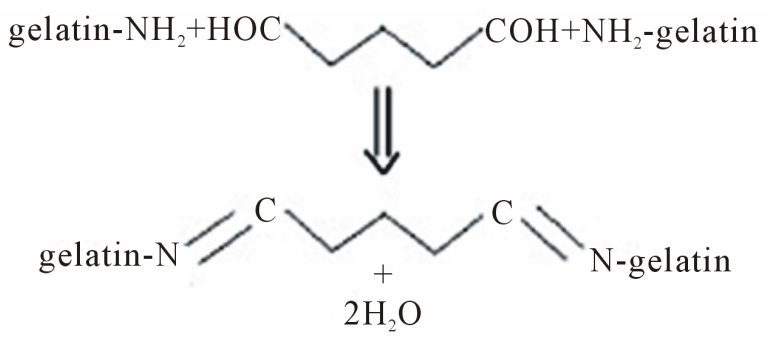

Moreover, the shrinkage of the fiber mats is responsible for the observed decrease in the size of inter-fibrouspores as well as the observed decrease in the thickness of the fiber mats and the observed increase in the diameters of the individual fibers.

Figure 6 shows the fiber morphologies of the samples before and after crosslinking. The fibrous form had been grossly preserved, however, due to the nanoscale size of the gelatin fibers: the coexistence of glutraldhyde (GTA) during crosslinking treatment had affected the fiber morphology to some extent. This is reflected by the fact that fibers at junctions were fused together forming bondings (the inset image of Figure 6(b)). It is clear that the range of diameters of the pores decreased with increasing the time of crosslinking (the inset image of Figure 6(c)).

\subsubsection{Release Characteristics of Silver}

Here, it is postulated that the as-formed $\mathrm{nAg}$ dissolved readily upon contact with the releasing medium, and that both the remnant and the dissolved $\mathrm{Ag}^{+}$ions were released into the medium during the release studies.

The release characteristics of $\mathrm{Ag}^{+}$ions from either 2 hrs or the 4 hrscrosslinkednAg-containing electrospun gelatin fiber mats samples, was investigated by the total immersion method in one of the releasing media (i.e., the buffer, and distilled water). The cumulative amount of $\mathrm{Ag}^{+}$ions released from these materials is reported in Figure 7 as the weight of $\mathrm{Ag}^{+}$ions released (in $\mathrm{mg}$ ) divided by the weight of the specimens (in g). Evidently, the cumulative amount of $\mathrm{Ag}^{+}$ions released from the samples in the buffer and distilled water occurred rather rapidly during the first $60 \mathrm{~min}$ after submersion in the releasing medium, and then increased gradually afterwards. 


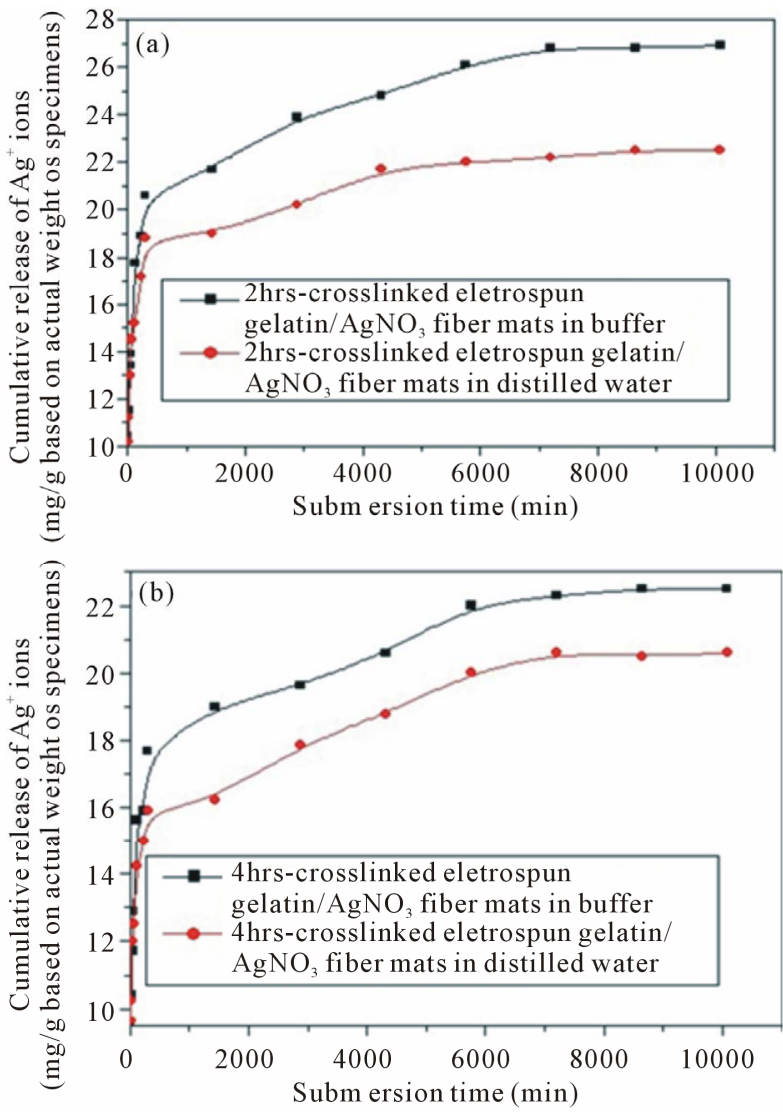

Figure 7. Cumulative release profiles of $\mathrm{Ag}^{+}$ions from 2and 4 hrs crosslinked nAg containing e-spun gelatin fiber mat specimens reported as the weight of $\mathrm{Ag}^{+}$ions released (in $\mathrm{mg}$ ) divided by the actual weight of specimens (in $\mathrm{g}$ ) in two types of releasing medium, i.e., (a) Buffer (pH 4); (b) Distilled water (pH 7), at room temperature of $25^{\circ} \mathrm{C}$.

Concerning the 2 hrs crosslinked nAg-containing espun gelatin fiber mat specimens, the cumulative amount of $\mathrm{Ag}^{+}$ions released from the specimens in distilled water was the same as that released in the buffer during the first 60 min of submersion, while that released from specimens in the buffer was increasingly greater afterwards. A similar trend was observed for the $4 \mathrm{hrs}$ crosslinked nAg-containing e-spun gelatin fiber mat specimens, in which the cumulative amount of $\mathrm{Ag}^{+}$ionsreleased from these specimens in distilled water was approximately the same as that amount released in the buffer during the first 60 min of submersion.

Between the two sample types, the cumulative amount of $\mathrm{Ag}^{+}$ions released from the $2 \mathrm{hrs}$ crossinked nAg-containing e-spun gelatin fiber mat specimens in any type of releasing medium was systematically greater than the amount released from the $4 \mathrm{hrs}$ crosslinked ones. This is possibly a result of the observed lower weight loss and water retention of the $4 \mathrm{hrs}$ crosslinked nAg-containing e-spun gelatin fiber mat specimens in comparison with those of their 2 hrscrosslinked counterparts.
Quantitatively, the cumulative amount of $\mathrm{Ag}^{+}$ions released from the $2 \mathrm{hrscrosslinkednAg-containing} \mathrm{e-spun}$ gelatin fiber mat specimens in the buffer increased from $\sim 13.9 \mathrm{mg} / \mathrm{g}$ of the specimens at $60 \mathrm{~min}$, to attain a final value of $\sim 26.9 \mathrm{mg} / \mathrm{g}$ of the specimens at day 7 . For the 4 hrs crosslinked nAg-containing e-spun gelatin fiber mat specimens, the amount of $\mathrm{Ag}^{+}$ions increased from $12.9 \mathrm{mg} / \mathrm{g}$ of the specimens at $60 \mathrm{~min}$ to $\sim 22.1 \mathrm{mg} / \mathrm{g}$ of the specimens at day 7. In distilled water, the cumulative amount of $\mathrm{Ag}^{+}$ions released from the $2 \mathrm{hrs}$ crosslinked nAg-containing e-spun gelatin fiber mat specimens increased from $\sim 14.5 \mathrm{mg} / \mathrm{g}$ of the specimens at $60 \mathrm{~min}$ to $~$ $22.2 \mathrm{mg} / \mathrm{g}$ of the specimens at day 7. For the $4 \mathrm{hrs}$ crosslinked nAg-containing e-spun gelatin fiber mat specimens, it increased from $\sim 12.5 \mathrm{mg} / \mathrm{g}$ of the specimens at $60 \mathrm{~min}$ to $\sim 20.1 \mathrm{mg} / \mathrm{g}$ of the specimens at day 7 .

The possible reason for the observed greater release of $\mathrm{Ag}^{+}$ions from both types of samples in buffer than in distilled water could be due to the protonation of the amino groups of gelatin. The $\mathrm{pH}$ value of the buffer medium was supposedly less than the isoelectric point (PI) of the type-A gelatin (i.e., PI $\approx 7-9$ ) used in this work [31].

\subsubsection{Antimicrobial Activity}

The potential use of nAg-containing e-spun gelatin fiber mats as functional wound dressings was assessed by observing their antibacterial activity (based on the disc diffusion method) against some common bacteria found on burn wounds: Escherichia coli, Pseudomonas aeroginosa, Staphylococcus aureus and Candida albicans. The activity of the neat e-spun gelatin fiber mats against these bacteria was used as a control. Since both the neat and the nAg-containing e-spun gelatin fiber mat specimens were crosslinked with GTA vapor to enhance their stability in an aqueous medium, the effect of glycine treatment on the neat e-spun gelatin fiber mats, and then Ag-containing e-spun gelatin fiber mat specimens to neutralize unreacted aldehyde groups of GTA [32].

It should be noted that the initial diameter of all the specimens was the same. However, in a wet condition, the specimens swelled, resulting in the observed dimensional change.

According to the results obtained, all of the crosslinked neat e-spun gelatin fiber mat specimens, with glycine treatment, showed no activity against the tested bacteria. For the nAg-containing specimens, inhibitory zones were evident.

Specifically, the antibacterial activity of the nAg-containing specimens, regardless of the sample type, was greatest against Staphylococcus aureus, followed by Escherichia coli, and Pseudomonas aeroginosa $\approx$ Candida albicans respectively Figure 8.

Feng et al. [33] demonstrated that, upon interacting 

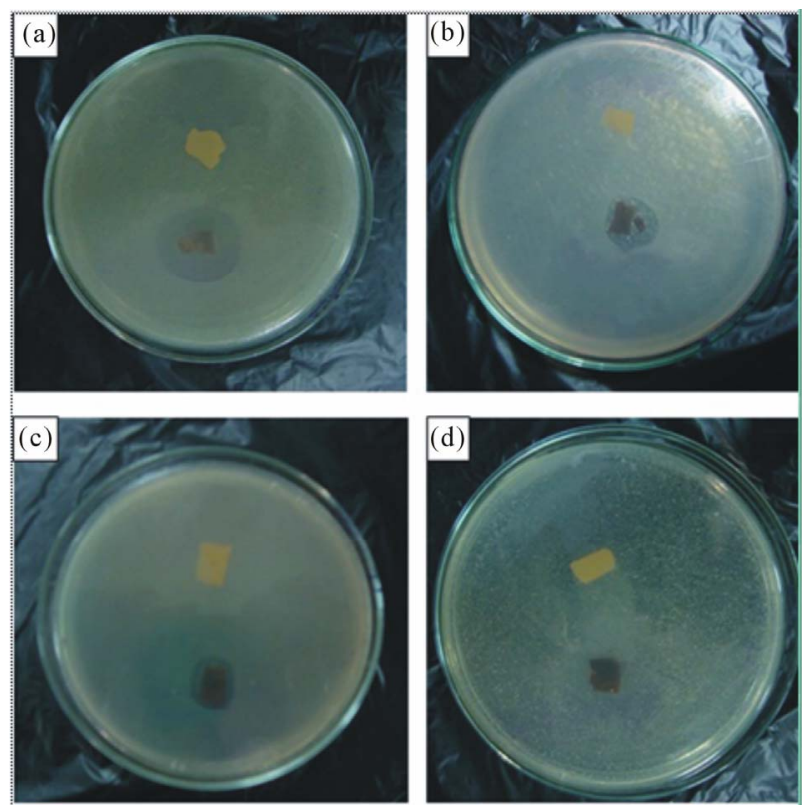

Figure 8. Antibacterial activity of the electrospun fiber mats from the base gelatin solution (upper) and the $12 \mathrm{~h}$-aged $\mathrm{AgNO}_{3}$-containing gelatin solution (lower), after having been crosslinked with glutaraldehyde against some common bacteria found on burn wounds. (a) Staphylococcus aureus; (b) Escherichia coli; (c) Pseudomonas aeroginosa and (d) Candida albicans.

with $\mathrm{Ag}^{+}$ions, both Escherichia coli and Staphylococcus aureus underwent a series of events that led to their demise. Specifically, they postulated that the bactericidal mechanism was based on the ability of Agions to bind with certain chemical functionalities of the cell wall, the cytoplasm and the nucleus. This causes DNA molecules to condense, the cytoplasmic membrane to detach from the cell wall, and the cell wall to become severely damaged [33]. A similar observation of the bactericidal effect of $\mathrm{nAg}$ and/or Agions has been reportedby [34,35].

\section{Conclusion}

Silver ions (Ag ions), known for their broad-spectrum antimicrobial activity, were reduced into silver nanoparticles (nAg) in a base gelatin (type A, porcine skin) solution $(50 \% \mathrm{w} / \mathrm{v}$ in $70 \mathrm{vol} \%$ acetic acid) containing 2.5 $\mathrm{wt} \% \mathrm{AgNO}_{3}$ (based on the weight of the gelatin powder). The presence of $\mathrm{nAg}$ in the $\mathrm{AgNO}_{3}$-containing gelatin solution was first realized after it had been aged for at least 12 hours. The average diameters of the as-formed $\mathrm{nAg}$ ranged between $\sim 2$ and $\sim 10 \mathrm{~nm}$. Both the neat gelatin and the 12 hours aged $\mathrm{AgNO}_{3}$-containing gelatin solutions were fabricated into ultrafine fibers by electrospinning $(20 \mathrm{kV} / 18 \mathrm{~cm})$. The average diameters of these fibers were in the range of $98-345$ and $154-390 \mathrm{~nm}$ respectively. Both the neat and the nAg-containing gelatin fiber mats were further crosslinked in order to im- prove their stability in an aqueous medium or a high humidity atmosphere by $2 \%$ glutaraldehyde (GTA) aqueous solution. Crosslinking not only caused the color of the cross-linked gelatinfibers to change, but also was responsible for the fusing and shrinking of the crosslinked fiber mats. The release characteristics of $\mathrm{Ag}$ ions from either the 2 or the 4 hrs-crosslinked nAg-containing e-spun gelatin fiber mats was investigated by the total immersion method in acetate buffer, and distilled water at room temperature. The cumulative release of $\mathrm{Ag}$ ions from the samples in the acetate buffer and distilled water occurred rather rapidly during the first 60 minutes after submersion in the releasing medium, and increased gradually afterwards. Lastly, the antibacterial activity of these materials (with glycinewashing) was greatest against $S$. aureus, followed by E. coli, P. aeroginosa $\approx$ Candida albicans respectively. The results confirmed the structure of silver nanoparticles containing nanofibers which could find important application in the biomedical field, e.g., as the wound dressings, due to the advantages of gelatin, and silver nanoparticles.

\section{Acknowledgements}

This work was supported by the Egyptian/German Project (GESP) and the In house project at the National Research Center.

\section{REFERENCES}

[1] D. N. Shier, J. L. Butler and R. Lewis, "Hole's Human Anatomy \& Physiology," 11th Edition, McGraw-Hill Higher Education, 2006.

[2] X. Wen, D. Shi and N. Zhang, "Applications of Nanotechnology in Tissue Engineering," In: H. S. Nalwa, Ed., Handbook of Nanostructured Biomaterials and Their Applications in Nanobiotechnology, American Scientific Publishers, 2005.

[3] B. Ding, H. Y. Kim, S. C. Lee, C. L. Shao, D. R. Lee, S. J. Park, et al., "Preparation and Characterization of a Nanoscalepoly (vinyl alcohol) Fiber Aggregate Produced by an Electrospinning Method," Journal of Polymer Science Part B: Polymer Physics, Vol. 40, No. 13, 2002, pp. 12611268.

[4] Z. M. Huang, Y. Z. Zhang, S. Ramakrishna and C. T. Lim, "Electrospinning and Mechanical Characterization of Gelatin Nanofibers," Polymer, Vol. 45, No. 15, 2004, pp. 5361-5368. http://dx.doi.org/10.1016/j.polymer.2004.04.005

[5] Y. Zhang, H. Ouyang, C. T. Lim, S. Ramakrishna and Z. M. Huang, "Electrospinning of Gelatin Fibers and Gelatin/PCL Composite Fibrous Scaffolds," Journal of Biomedical Materials Research Part B: Applied Biomaterials, Vol. 72B, No. 1, 2005, pp. 156-165.

http://dx.doi.org/10.1002/jbm.b.30128

[6] M. Li, Y. Guo, Y. Wei, A. G. MacDiarmid and P. I. Lelkes, "Electrospinningpolyaniline Contained Gelatin Nan- 
ofibers for Tissue Engineering Applications," Biomaterials, Vol. 27, No. 13, 2006, pp. 2705-2715. http://dx.doi.org/10.1016/j.biomaterials.2005.11.037

[7] M. Li, M. J. Mondrinos, M. R. Gandhi, F. K. Ko, A. S. Weiss and P. I. Lelkes, "Electrospun Protein Fibers as Matrices for Tissue Engineering," Biomaterials, Vol. 26, No. 30, 2005, pp. 5999-6008. http://dx.doi.org/10.1016/j.biomaterials.2005.03.030

[8] S. Young, M. Wong, Y. Tabata and A. G. Mikos, "Gelatin as a Delivery Vehicle for the Controlled Release of Bioactive Molecules," Journal of Controlled Release, Vol. 109, No. 1-3, 2005, pp. 256-274. http://dx.doi.org/10.1016/j.jconrel.2005.09.023

[9] A. Jongjareonrak, S. Benjakul, W. Visessanguan, T. Prodpran and M. Tanaka, "Characterization of Edible Films from Skin Gelatin of Brownstripe Red Snapper and Bigeye Snapper," Food Hydrocolloids, Vol. 20, No. 4, 2006, pp. 492-501.

http://dx.doi.org/10.1016/j.foodhyd.2005.04.007

[10] F. R. M. Huss, J. P. E. Junker, H. Johnson and G. Kratz, "Macroporousgelatine Spheres as Culture Substrate, Transplantation Vehicle, and Biodegradable Scaffold for Guided Regeneration of Soft Tissues. In Vivo Study in Nude Mice," Journal of Plastic, Reconstructive \& Aesthetic Surgery, Vol. 60, No. 5, 2007, pp. 543-555.

http://dx.doi.org/10.1016/j.bjps.2005.10.031

[11] J. Vandervoort and A. Ludwig, "Preparation and Evaluation of Drug-Loaded Gelatin Nanoparticles for Topical Ophthalmic Use," European Journal of Pharmaceutics and Biopharmaceutics, Vol. 57, No. 2, 2004, pp. 251-261. http://dx.doi.org/10.1016/S0939-6411(03)00187-5

[12] Y. Tabata, S. Hijikata and Y. Ikada, "Enhanced Vascularization and Tissue Granulation by Basic Fibroblast Growth Factor Impregnated in Gelatin Hydrogels," Journal of Controlled Release, Vol. 31, No. 2, 1994, pp. 189199. http://dx.doi.org/10.1016/0168-3659(94)00035-2

[13] T. Y. Liu, S. H. Hu, K. H. Liu, D. M. Liu and S. Y. Chen, "Preparation and Characterization of Smart Magnetic Hydrogels and Its Use for Drug Release," Journal of Magnetism and Magnetic Materials, Vol. 304, 2006, pp. 397399.

[14] J. Liu, D. Meisner, E. Kwong, X. Y. Wu and M. R. Johnston, "A Novel Trans-Lymphatic Drugdelivery System: Implantable Gelatin Sponge Impregnated with PLGAPaclitaxel Microspheres," Biomaterials, Vol. 28, No. 21, 2007, pp. 3236-3244. http://dx.doi.org/10.1016/j.biomaterials.2007.03.022

[15] R. Fukae, A. Maekawa and O. Sangen, "Gel-Spinning and Drawing of Gelatin," Polymer, Vol. 46, No. 25, 2005, pp. 11193-11194. http://dx.doi.org/10.1016/j.polymer.2005.10.001

[16] J. B. Wright, K. Lam, A. G. Buret, M. E. Olson and R. E. Burrell, "Early Healing Events in a Porcine Model of Contaminated Wounds: Effects of Nanocrystalline Silver on Matrix Metalloproteinases, Cell Apoptosis, and Healing," Wound Repair and Regeneration, Vol. 10, No. 3, 2002, pp. 141-151.

http://dx.doi.org/10.1046/j.1524-475X.2002.10308.x

[17] S. A. Jones, P. G. Bowler, M. Walker and D. Parsons,
"Controlling Wound Bioburden with a Novel Silver-Containing Hydrofiber ${ }^{\circledR}$ Dressing," Wound Repair and Regeneration, Vol. 12, No. 3, 2004, pp. 288-294. http://dx.doi.org/10.1111/j.1067-1927.2004.012304.x

[18] C. L. Gallant-Behm, H. Q. Yin, S. Jui, J. P. Heggers, R. E. Langford, M. Olson, et al., "Comparison of in Vitro Disc Diffusion and Time Kill-Kinetic Assays for the Evaluation of Antimicrobial Wound Dressing Efficacy," Wound Repair and Regeneration, Vol. 13, No. 4, 2005, pp. 412421. http://dx.doi.org/10.1111/j.1067-1927.2005.130409.x

[19] D. J. Leaper, "Silver Dressings: Their Role in Wound Management," International Wound Journal, Vol. 3, No. 4, 2006, pp. 282-294.

http://dx.doi.org/10.1111/j.1742-481X.2006.00265.x

[20] C. K. Field and M. D. Kerstein, "Overview of Wound Healing in a Moist Environment," The American Journal of Surgery, Vol. 167, No. 1, 1994, pp. 2-6. http://dx.doi.org/10.1016/0002-9610(94)90002-7

[21] D. H. Reneker and A. L. Yarin, "Electrospinning Jets and Polymer Nanofibers," Polymer, Vol. 49, No. 10, 2008, pp. 2387-2425. http://dx.doi.org/10.1016/j.polymer.2008.02.002

[22] F. Tofoleanu, T. B. Mindru, F. Brinza, N. Sulitanu, I. C. Sandu, D. Raileanu, et al., "Electrospun Gelatin Nanofibers Functionalized with Silver Nanoparticles," Journal of Optoelectronics and Advanced Materials, Vol. 10, No. 12, 2008, pp. 3512-3516.

[23] R. Yoskan and S. Chirachanchai, "Silver Nanoparticles Dispersing in Chitosan Solution: Preparation by GammaRay Irradiation and Their Antimicrobial Activities," Materials Chemistry and Physics, Vol. 115, No. 1, 2009, pp. 296-302.

http://dx.doi.org/10.1016/j.matchemphys.2008.12.001

[24] C. S. Ki, D. H. Baek, K. D. Gang, K. H. Lee, I. C. Um and Y. H. Park, "Characterization of Gelatin Nanofiber Prepared from Gelatin-Formic Acid Solution," Polymer, Vol. 46, No. 14, 2005, pp. 5094-5102.

http://dx.doi.org/10.1016/j.polymer.2005.04.040

[25] H. Akin and N. Hasirci, "Preparation and Characterization of Crosslinked Gelatinmicrospheres," Journal of Applied Polymer Science, Vol. 58, No. 1, 1995, pp. 95-100. http://dx.doi.org/10.1002/app.1995.070580110

[26] A. Bigi and G. Cojazzi, "Mechanical and Thermal Properties of Gelatin Films at Different Degrees of Glutaraldehyde Crosslinking," Biomaterials, Vol. 22, No. 8, 2001, pp. 763-768. http://dx.doi.org/10.1016/S0142-9612(00)00236-2

[27] H. Nagahama, H. Maeda, T. Kashiki, R. Jayakumar, T. Furuike and H. Tamura, "Preparation and Characterization of Novel Chitosan/Gelatin Membranes Using Chitosan Hydrogel," Carbohydrate Polymers, Vol. 76, No. 2, 2009, pp. 255-260. http://dx.doi.org/10.1016/j.carbpol.2008.10.015

[28] A. Yiwei, Y. Yunxia, Y. Shuanglong, D. Lihua and D. Guorong, "Preparation of Spherical Silver Particles for Solar Cell Electronic Paste with Gelatin Protection Mater," Materials Chemistry and Physics, Vol. 104, No. 1, 2007, pp. 158-161. http://dx.doi.org/10.1016/i.matchemphys.2007.02.102 
[29] Y. Z. Zhang, J. Venugopal, Z. M. Huang, C. T. Lim and S. Ramakrishna, "Crosslinking of the Electrospun Gelatin Nanofibers," Polymer, Vol. 47, No. 8, 2006, pp. 29112917. http://dx.doi.org/10.1016/i.polymer.2006.02.046

[30] L. H. H. Olde Damink, P. J. Dijkstra, M. J. A. van Luyn, P. B. van Wachem, P. Nieuwenhuis and J. Feijen, "Glutaraldehyde as a Crosslinking Agent for Collagen-Based Biomaterials," Journal of Materials Science: Materials in Medicine, Vol. 6, No. 8, 1995, pp. 460-472. http://dx.doi.org/10.1007/BF00123371

[31] D. Yang, Y. Li and J. Nie, "Preparation of Gelatin/PVA Nanofibers and Their Potential Application in Controlled Release of Drugs," Carbohydrate Polymers, Vol. 69, No. 3, 2007, pp. 538-543. http://dx.doi.org/10.1016/j.carbpol.2007.01.008

[32] K. S. Rho, L. Jeong, G. Lee, B. M. Seo, Y. J. Park, S. D. Hong, et al., "Electrospinning of Collagen Nanofibers: Effects on the Behavior of Normal Human Keratinocytes and Early-Stage Wound Healing," Biomaterials, Vol. 27,
No. 8, 2006, pp. 1452-1461.

http://dx.doi.org/10.1016/j.biomaterials.2005.08.004

[33] Q. L. Feng, J. Wu, G. Q. Chen, F. Z. Cui, T. N. Kim and J. O. Kim, "A Mechanistic Study of the Antibacterial Effect of Silver Ions on Escherichia Coli and Staphylococcus Aureus," Journal of Biomedical Materials Research, Vol. 52, No. 4, 2000, pp. 662-668. http://dx.doi.org/10.1002/1097-4636(20001215)52:4<662 $\because$ AID-JBM10>3.0.CO;2-3

[34] J. R. Morones, J. L. Elechiguerra, A. Camacho, K. Holt, J. B. Kouri, J. T. Ramírez, et al., "The Bactericidal Effect of Silver Nanoparticles," Nanotechnology, Vol. 16, No. 10, 2005, pp. 2346-2353.

http://dx.doi.org/10.1088/0957-4484/16/10/059

[35] S. Shrivastava, T. Bera, A. Roy, G. Singh, P. Ramachandrarao and D. Dash, "Characterization of Enhanced Antibacterial Effects of Novel Silver Nanoparticles," Nanotechnology, Vol. 18, No. 22, Article ID: 225103. 